Humanist Studies \& the Digital Age, 6.1 (2019)

ISSN: 2158-3846 (online)

http://journals. oregondigital.org/hsda/

DOI: $10.5399 /$ uo/hsda.6.1.1

(cc) BY-ND

\title{
Pierre Lévy and the Future of Internet
}

\section{Massimo Lollini}

Semantic Metadata, Humanist Computing and Digital Humanities, opens with an important interview with Pierre Lévy that reconstructs the key moments of his philosophical vision of the internet, and the World Wide Web, up to his most recent and highly innovative proposal of the Information Economy MetaLanguage (IEML).

If we were to identify a common element to the different aspects of his thought, we could say that this element consists in the search for the actual living unity of human knowledge despite human scattering in space and time. This element was already present in his idea of collective intelligence theorized in 1994 book, Intelligence collective: Pour une anthropologie du cyberspace (Collective Intelligence: Mankind's Emerging World in Cyberspace); and has undergone a precise theoretical elaboration in World Philosophie: le marché, le cyberespace, la conscience (2000).

The latter book offers a philosophical synthesis that goes far beyond the partial reflections that remain focused on discrete aspects of the so-called technological revolution advanced by the Internet and the WWW that scholars often analyze by paying almost exclusive attention to individual devices, on-line reading habits, special computing solutions or social networking practices. The result of these partial analyses often leads critics to condemn the technological revolution in its entirety. Pierre Lévy's work is an antidote to these unilateral, quite often apocalyptic, positions and offers the theoretical tools to elaborate a vision of humanity critically unified in and by the space of knowledge.

The interview reconstructs Lévy's vision from its origins in a reflection on medieval Neoplatonic thought, based on a top-down model, to the bottom-up model of collective intelligence that he considers as the engine of human development toward the unification of the planet. In his view, the unification of humanity also includes that of the planet through the digital representation of the physical world that will have beneficial effects in the search for sustainable development.

Other topics touched on in the interview concern the relationship between the Internet and social media, the problem of "fake news," and the phenomenon of so-called "post-truth," both in the 1930s, when the prevailing medium was radio, and in the time of the Internet characterized by the rise of social media. The problem of new forms of memory needed by collective intelligence at the time of the Internet is also briefly touched on in the interview.

Much space is devoted to the future of the Internet, especially in the second part of the interview where Lévy introduces his most recent project, the IEML. Regarding the future of the Internet Lévy shares the recent concerns advanced by Tim Berners-Lee, the creator of the WWW. Lévy points out that at a time when more than 60 percent of the human population is connected to the Internet, and 
all information transmitted is reduced to digital data, it becomes crucial for users to be sovereign over their data, which are currently expropriated by the giants of Internet like Google and Facebook.

However, the proposal to create the IEML derives from another need, that of augmenting our collective categorization power and developing new symbolic systems suitable for the digital medium. The IEML is essentially a language, a sophisticated system of semantic metadata that will allow humans to do much more refined conceptual research than is possible today. In short, the IEML aspires to be a new layer of a universal addressing system, at a time when, for the first time in human history, the human species is growing a universally interconnected common memory where ubiquitous data can be accessed and transformed by automatic symbol manipulators.

The new universal addressing system is called Uniform Sematic Locator (USL) via semantic tags; it is conceived as a new layer on top of the actual interconnection between documents via the Web and the URLs. IEML promotes interconnection between ideas and offers a semantic space capable to augment collective intelligence by bringing together the humanistic disciplines and the social sciences.

Lévy explains in detail the various aspects of his proposal including its new anthropological space, the "space of knowledge," and in the Appendix to the interview he offers some very interesting paradigms of morphemes for the IEML.

In the "Interventions" section our journal features an important reflection by Dino Buzzetti on the distinction between Humanities Computing and Digital Humanities. The essay, originally published in Italian, critically supports the rationales behind Humanities Computing, characterized by a primary interest in methodological issues and their epistemological background. Buzzetti reconstructs accurately the history of this idea starting from the seminal works of scholars like JeanClaude Gardin, who underlined the need for an awareness that computation applied to the humanities requires both representation (data structures), and information processing (algorithms).

Buzzetti argues that this awareness has been lost with the advent of Digital Humanities that have focused on the pure representation of research data, losing sight of their cognitive and algorithmic processing. In this perspective, the Digital Humanities, with proposals like the Textual Encoding Initiative (TEI), has become a document community taking care of the automation of the visualization and printing processes of the documents, setting aside the vision of a community dedicated instead to the design of archives structured data, the database community.

While not expounding the sort of philosophical and phenomenological vision that characterizes Lévy's approach, Buzzetti's intervention goes well with the ideas expressed by Lévy in stressing the cognitive value of Humanities Computing and the new design dimension of culture within the socalled Semantic Web. Common to the two scholars, albeit from different perspectives, is also the search for formal models that allow a methodological convergence between the humanities and the sciences, between the predominantly objective methods of the natural sciences and the prevalently subjective ones of literary or discursive production in general.

Buzzetti's analysis also includes the markup used for the digital representation of the text that he considers simultaneously "representation" and "representation of the representation." After all, he argues, the cyclic and self-referential nature of the discursive process itself leads to a theoretical 
perspective which, following scholars such as Varela and Merleau-Ponty, excludes the absolute separation between the subject and the object, between the observer and the observed.

The recognition of the unavoidable relationship between the observer and the systems observed is not limited to the human sciences but extends to the field of physical and biological sciences. For this reason, Buzzetti concludes, the paradigm of self-referentiality seems to open a new perspective of convergence between the methods of natural sciences and the methods of the human sciences characterized by the implementation of a computational model of the discursive processes proper to scientific constructions of the humanities.

The three projects that are introduced in the third part of the journal respond differently to the theoretical solicitations presented in the first two sections. Following the categories of Pierre Lévy, we should say that, even if in a different way, all three projects are the product of a collective intelligence and at the same time contribute to expand the knowledge of a physical territory (in the case of Noisemakers! and of The Dialogues Bioregional Project) or of a literary tradition (in the case of \#Laura speaks), making the process of their digital processing transparent.

On the other hand, if instead we wanted to consider these projects from the point of view of the categories proposed by Buzzetti, we should indicate that they belong above all to the realm of the Digital Humanities and only partially to that of Humanist Computing. The prevailing interest of these projects is in fact the representation and remediation of data belonging to the physical and/or cultural world. Furthermore, all three are highly invested in extending the reach of the digital humanities beyond the borders of academia and into the public realm.

Noisemakers! Putting the Analog in Digital Humanities by Serena Ferrando and Mark Wardecker describes an innovative collaborative and interdisciplinary project whose main purpose is to create concrete opportunities for students to participate in the "real" world and engage with the materiality of noise and its manifestations by interacting with the soundscape through interactive and multisensory practices. Noisefest! is centered around Waterville, a small Maine town, and rooted in the sounds and noise of its streets. It comprises a Virtual Reality tour, soundwalks and remixes, a 2D laser cut geographical map with Arduino controllers, and a Futuristic noise intoner. At the intersection of theory and experiential learning, this project help students developing the ability to portray and "play" the city of as a musical instrument to draw attention to the city as a multilayered sound system.

The Dialogues Bioregional Project: Landscape Ecology in Central Italy from the Sixth Century to the Present by Damiano Benvegnù, outlines the "Dialogues Bioregional Project," a digital, interdisciplinary interface on Italian landscape ecology which promotes dialogues between scientists and humanists as well as provide a modeling tool for environmental and cultural awareness. Shaped around the "Dialogues" of Pope Gregory I, this digital humanities project explores continuities and discontinuities between the socio-political and ecological history of a specific section of Italian territory, a set of multidisciplinary environmental narratives (from c. $600 \mathrm{AD}$ to the present), and local communities. Ultimately, this project will encourage communities to re-approach their natural settings in ecological terms. 
\#LauraSpeaks: Remediations of Pellegra Bongiovanni's "Risposte" by Elisa Briante, Marena Lear, and Gerardo Pisacane, is a project meant to enrich the database of the Oregon Petrarch Open Book with a translation and remediation of Pellegra Bongiovanni's Risposte di Madonna Laura alle rime di Messer Francesco Petrarca, in nome della medesima (1763). Each of the three authors contributes an original essay. Elisa Briante's "Voicing Laura: Pellegra Bongiovanni's challenge" illustrates Bongiovanni's importance within the realm of Petrarchism, Marena Lear's “A Living Text: Pellegra Bongiovanni's Risposte and Theories of Remediation" discusses the translation of Bongiovanni's work from Italian to English and the creation of the "Twitterature" version of the text. Finally, Gerardo Pisacane's "Francesco, You've Got Mail - the Movie. Literary criticism through cinema" analyses the remediation of Bongiovanni's Risposte into the film medium. The authors also investigate the theoretical premises of digital remediation and the role that hypertext plays in multiplying opportunities for meaningmaking and in enriching the act of reading and writing.

In closing these brief introductory notes to the volume on Semantic Metadata, Humanist Computing and Digital Humanities, I would like to invite the authors of this issue and the other authors who over the years have contributed to Humanist Studies and the Digital Age to propose the topic of the next issue of the journal, to be published in 2021. The invitation is extended to all readers of the journal who are passionate about our analysis of the fate of writing and reading in the Internet age. Already in this issue the interview with Pierre Lèvy adds a philosophical and phenomenological dimension to this exciting theme 